\title{
Integrated High Frequency Coaxial Transformer Design Platform Using Artificial Neural Network Optimization and FEM Simulation
}

\author{
H. Li, W. Water, B. Zhu and J. Lu \\ Queensland Micro- and Nanotechnology Centre, School of Engineering, Griffith University, Nathan, 4111, Australia \\ jeffrey.li@griffithuni.edu.au
}

\begin{abstract}
Designing a high frequency (HF) power transformer is a complicated task due to its multiple interrelation design procedures, large number of variables and other relevant factors. Traditional transformer design relies on manual paper work and personal experience, which requires engineering design man-hours and long delivery cycles. In this paper, a developed transformer computer design environment is addressed. It helps engineers to automatically model, simulate and optimize transformer design using an artificial neural network (ANN) algorithm and the finite element method (FEM), and delivers a reliable design result. By utilizing the proposed platform, an $8 \mathrm{~kW}$ coaxial transformer is successfully designed, tested and manufactured.
\end{abstract}

Index Terms - artificial neural network, finite element method, high frequency transformer, transformer design platform.

\section{INTRODUCTION}

$\mathrm{T}$ he traditional design methodology of a transformer is rarely achieved from scratch. In most cases, the transformer design procedure starts from adaption, or modification of an existing and proven transformer model. The design of an actual new transformer is time consuming and expensive. In order to shorten the development cycle and increase product performance, there is now an increasing demand for a software platform to assist engineers in transformer design.

In past literatures, an artificial neural network (ANN) was employed in the low frequency transformer design area. For most cases, the ANN was only used to detect and analyze the transformer's faults [1]-[3] or predict its performance [4] [5]. In addition, the decision trees method, along with ANNs, could easily solve the winding material selection problem [6] [9]. However, once the operating frequency of magnetics rises to $\mathrm{HF}$, traditional methods are no longer reliable due to the accompanying HF effects. For this reason, numerical techniques are applied to deal with HF problems. Among these numerical techniques, the finite element method (FEM) is very popular and evidence of its effectiveness is seen in many publications [6]- [8].

In this paper, a new integrated transformer computer design environment is presented using an accumulated transformer database and an ANN to automatically design and optimize a $\mathrm{HF}$ transformer structure. In addition, the FEM solver is implemented to solve the numerical problem. To validate and demonstrate the effectiveness of the introduced design platform, an $8 \mathrm{~kW}$ high frequency and high power density coaxial transformer (HFCT) has been used as a case study example throughout this paper. The transformer has an operating frequency range of $100 \mathrm{kHz}$ to $300 \mathrm{kHz}$, and can easily scaled for power rating range between $1 \mathrm{~kW}$ and $20 \mathrm{~kW}$ due to its symmetrical winding structures [10].

\section{BASIC CONCEPT OF INTEGRATED DESIGN PLATFORM}

\section{A. Conventional HF Transformer Design Methodology}

The traditional design technique is based on magnetic circuit

Manuscript received May 22, 2014

Corresponding author: H. Li (e-mail: Jeffrey.li@griffithuni.edu.au).

Digital Object Identifier inserted by IEEE theory; thus, parameters such as the window size, the core dimension and air-gap of transformers can be easily obtained. The transformer universal voltage equation is written as:

$$
E_{r m s}=K_{a} \cdot f \cdot N \cdot B \cdot A_{\text {core }}
$$

where (1) describes the relationship of root mean square (RMS) voltage with supplied frequency $f$, winding turns $N$, crosssectional area $A_{\text {core }}$ of the transformer core and constant $K_{a}$; where for square wave supply, $K_{a}=4$, sine wave supply, $K_{a}=$ 4.44 .

The traditional method on magnetic design is relatively simple and reliable, especially for low frequency magnetics. However, the "magnetics issue" at HF becomes a bottleneck of the traditional method at high frequencies. Other than that, the repeated and redundant calculation process is another concern. The success of traditional methods on magnetic design therefore depends largely on the experience of engineers.

\section{B. ANN Concept and Methodology}

An artificial neural network has many benefits compared with the human brain. It is nearly one million times faster in terms of computing speed, it can process in parallel and provide interconnection storage. Moreover, it has a good control mechanism with high fault tolerance. Equation (2) shows a McCulloch-Pitts model [11]:

$$
\boldsymbol{y}_{j}=\sum \boldsymbol{w}_{i j} \boldsymbol{x}_{\boldsymbol{i}}-\boldsymbol{\theta}_{j}
$$

where $\theta$ is the neuron's activation threshold, $w_{i j} x_{i}$ is the input. By training the ANN, it can "learn" the potential relationship between selected input/output parameters. Thus, the ANN can assist engineers in determination of magnetic circuit design, material selection and even structural design.

\section{Numerical Computation of Magnetic Field}

To evaluate the transformer's performance, FEM based electromagnetic packages can be used to conduct the analysis. The FEM solver has demonstrated its effectiveness in many research publications. To analyse the eddy current and magnetic fields of the HF magnetics, the detailed magnetic field properties for the HF integrated magnetics were used to 
facilitate the design of a low loss winding and high efficiency structure. The mathematical problem can be formulated as a sinusoidal quasi-static eddy current problem, which was derived from Maxwell's equations. The equations are described in terms of the complex magnetic vector potential $A$ and an electrical complex scalar potential $\phi$,

$$
\nabla \times \frac{1}{\mu} \nabla \times A-\nabla\left(\frac{1}{\mu} \nabla \cdot A\right)+j \omega \sigma(A+\nabla \phi)=J_{S}
$$

where; $\mu$ is the permeability, $\sigma$ is the conductivity, $\omega$ is the angular frequency and $J_{S}$ is the excitation current density. It is difficult to solve the quasi-static eddy current problem mathematically with accurate results. Moreover, the use of Galerkin's method brings the approximated result more accurate. Thus, equation (3) is rewritten as shown in (4).

$$
G=[S]\{A\}+[M]\{A\}-[K]
$$

where the matrix $[\mathrm{S}]$ is the global coefficient matrix. $[\mathrm{M}]$ is the time harmonic matrix and $\mathrm{G}$ is the weighted residual. $[\mathrm{K}]$ is current density related matrix.

\section{IMPLEMENTATION OF AN ANN IN A DESIGN PLATFORM}

\section{A. Integrated Design Platform Structure}

The system follows the procedures as shown in Fig. 1. It contains magnetic circuit design, structure design and FEM simulation.

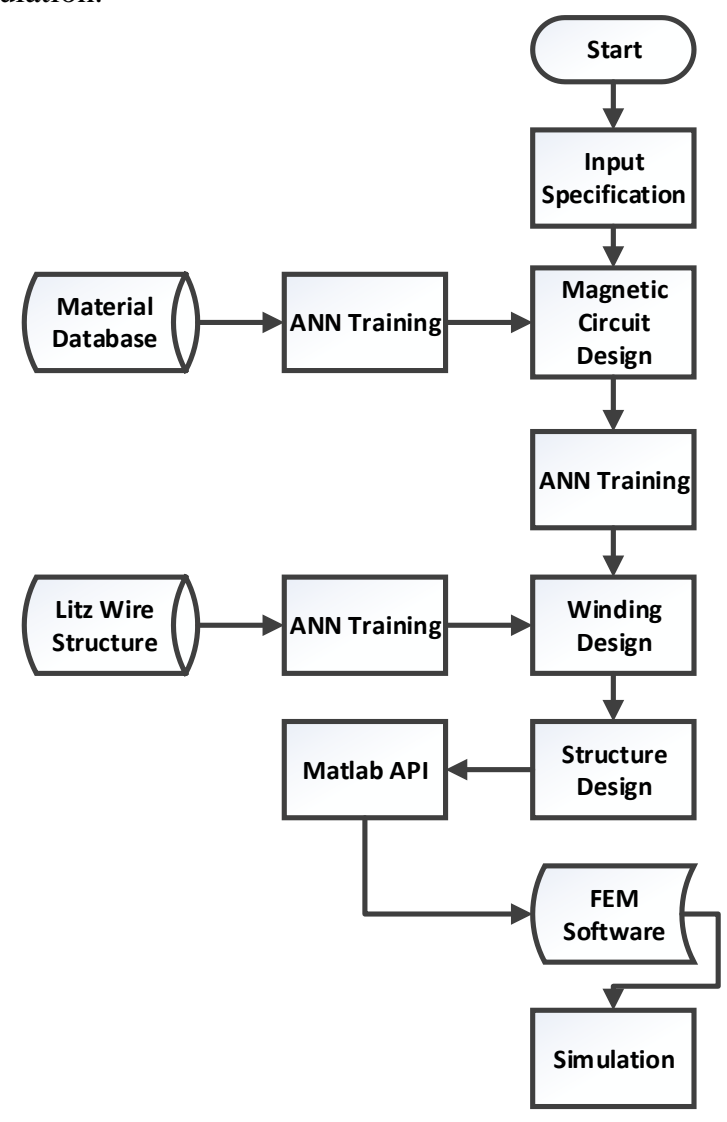

Fig. 1 Integrated Design Platform Structure
In order to simplify the design procedure, the platform only requires engineers to input several key parameters, such as transformer power, voltage, desired working frequency and the number of ring cores. After the engineer enters design parameters, the platform will calculate the magnetic circuit, communicate with the material database and select one material which is according to the working frequency. The litz wire structure database provides information on the winding design procedure by considering the power loss and core window size.

To communicate with FEM solver, MATLAB API can generate a structure matrix and pass it to the FEM solver. The FEM simulation provides a FEM result to help with the analysis of the eddy current and magnetic flux.

ANN in the platform is used to provide suggestion selected result to the user, such as material selection and winding structure. It is used to assist engineer designing the coaxial transformer.

\section{B. ANN with System Diagram}

Based on the ANN "black box concept" [5], the ANN system diagram is shown in Fig. 2. The ANN algorithm in the present study is under so-called supervised training methodology. The network gains the data from the database and determines the corresponding target $Z$. The engineer engages the design process as supervisor and provides target result ZT based on the FEM solver result. The differences iteratively between $\mathrm{Z}$ and ZT can be reduced by evaluating the ZT and adjusting the weights. In this platform, between each design procedure, the ANN engages the design and provides the selection result to the engineer.

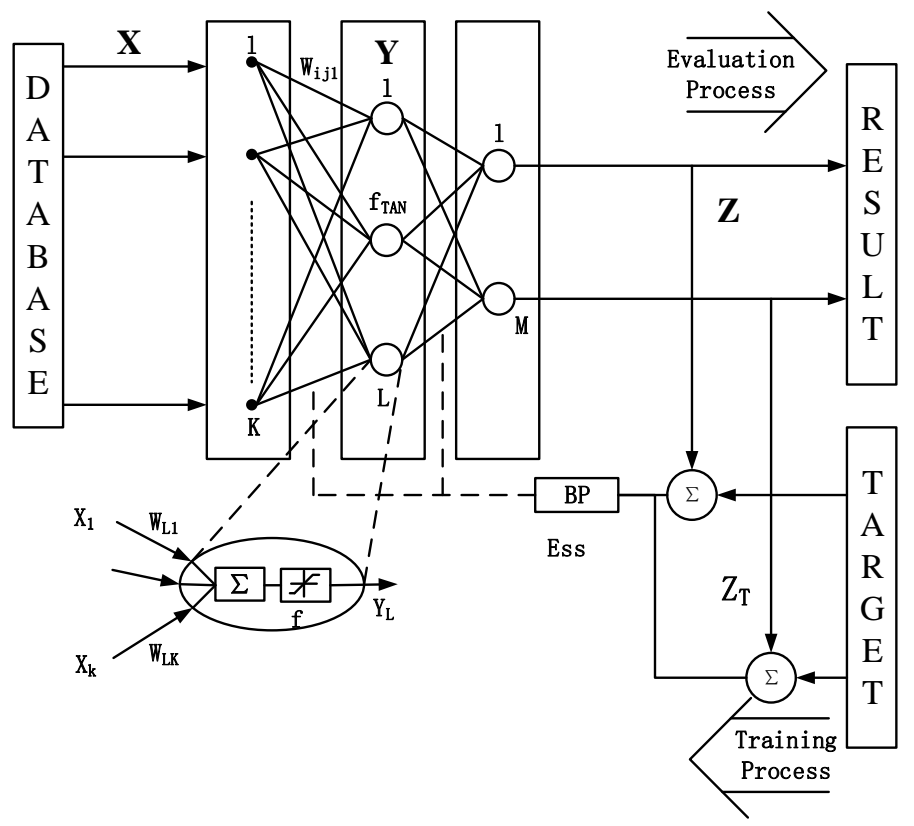

Fig. 2 ANN Implementation Diagram in a Platform

\section{Winding Structure Database}

There is a number of wiring configurations can be chosen, and sometimes selecting the best combination could be complicated and redundant. Thus, the introduced platform is able to automatically simulate and select the most sufficient option according to the requirements and ANN result. For 
example, generalized power losses versus the wire numbers is shown in Fig. 3, in which the upper limit of wire numbers is subject to the available transformer core window area.

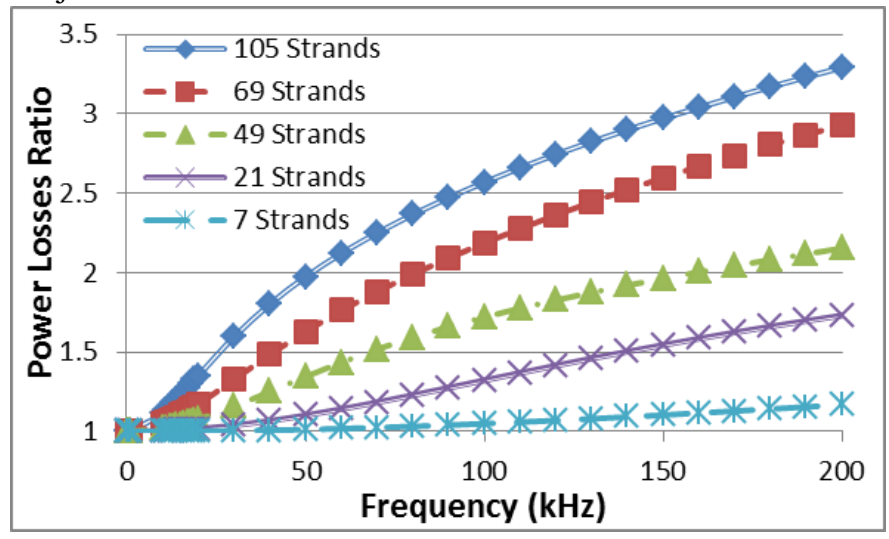

Fig. 3 Power losses comparison of multi-strand Litz wires (normalized with DC losses)

\section{IMPLEMENTATION OF DESIGN PLATFORM OF $8 \mathrm{KW}$ TRANSFORMER}

The required power rating specification of the example HFCT is tabulated in Table I 错误!未找到引用源。. The prototype transformer was built with the turn ratio of 1:1 for use as the isolation transformer. The unique coaxial winding structure can easily fulfil the transformer requirements of low eddy current loss, high power density, low leakage inductance and high electromagnetic compatibility. An operating frequency of between $100 \sim 300 \mathrm{kHz}$ was chosen because of concerns on the overall size and the electromagnetic interference (EMI) issue. A higher operating frequency effectively reduces the magnetizing current and size of the designed transformer. However, an increase in the operating frequency results in a higher AC winding loss, as well as the HF noise propagation problem. Furthermore, the prototype HFCT has power efficiency greater than $99 \%$, which has been experimentally verified under full-load conditions.

TABLE I

THE SPECIFICATION OF AN $8 \mathrm{KW}$ HFCT PROTOTYPE

\begin{tabular}{lcc}
\hline \hline Operation Frequency & $\mathrm{f}$ & $100 \mathrm{kHz}-300 \mathrm{kHz}$ \\
\hline Input voltage & $\mathrm{V}_{1}$ & $400 \mathrm{~V}(\mathrm{dc})$ \\
Input current & $\mathrm{I}_{1}$ & $20 \mathrm{~A}$ \\
Output voltages & $\mathrm{V}_{2}$ & $400 \mathrm{~V}(\mathrm{dc})$ \\
Power range & $\mathrm{P}$ & $8 \mathrm{k} \mathrm{VA}$ \\
Turn ratio & $\mathrm{N}_{1} / \mathrm{N}_{2}$ & 1 \\
Voltage ratio & $\mathrm{V}_{1} / \mathrm{V}_{2}$ & 1 \\
Power efficiency & & $>99 \%$ \\
Current density & $\mathrm{J}_{1}=\mathrm{J}_{2}$ & $5.25 \mathrm{~A} / \mathrm{mm} 2$ \\
Magnetic ferrite & & $\mathrm{Neosid} \mathrm{F} 44$ \\
Initial permeability & & $1900 \pm 20 \%$ \\
Temperature & $\mathrm{T}_{\max }$ & $60^{\circ} \mathrm{C}$ \\
\hline
\end{tabular}

\section{A. Structure Design Based on the Conventional Method}

The platform can list several different structure design results based on the working frequency, turns and the size of the ring core magnetic cross area. The engineer can obtain and compare different designs, then select a desired structure based on the requirements, as shown in Fig. 4. The figure gives a predicted results list for the transformer ring core structures. The ANN in this step will be trained by obtain the user selection.

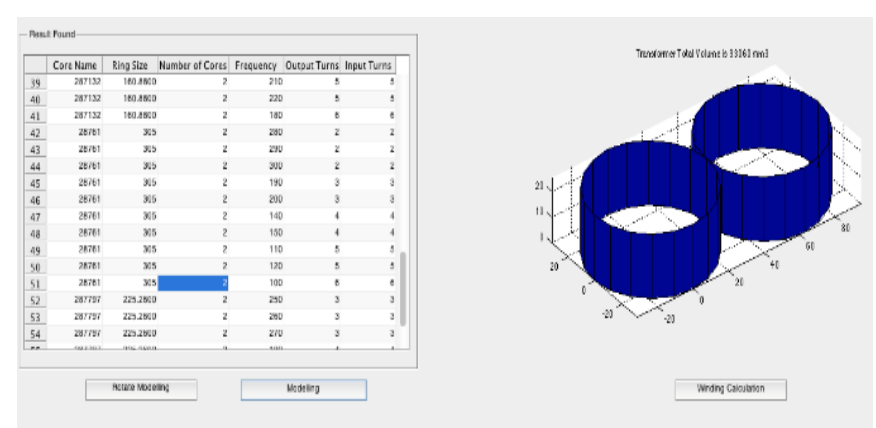

Fig. 4 Core combination selection example of the introduced platform

\section{B. Structure Optimization Based on an ANN}

The litz wire structure is considered in this step to generate a winding structure. Due to the factors of working frequency and the number of litz wires, the power loss can be different. The platform has generated a database which contains handers of different litz wire structures with a comparison figure. Shown in the Fig.5, a suggestion structure is selected from database by ANN.
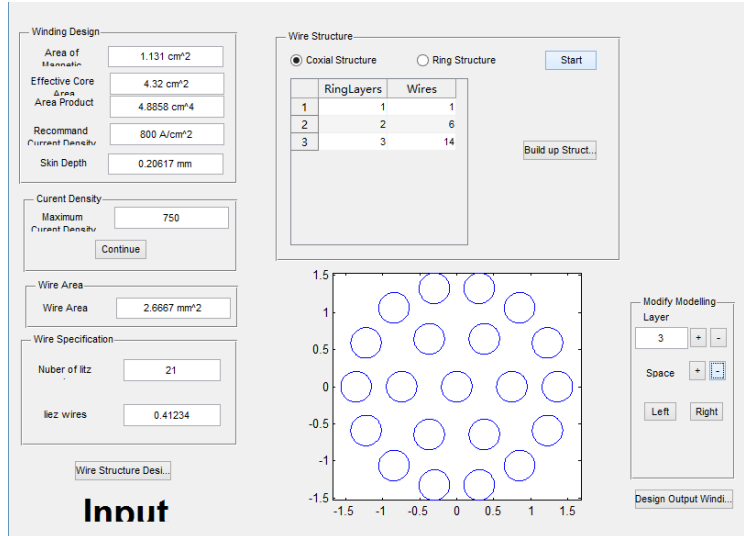

Fig. 5 Fine tuning of wire numbers and configurations

C. Structure Modelling \& Simulation of Magnetic Field

To simulate the transformer in an electromagnetic field, the platform firstly generates a coaxial transformer structure and allows the engineer to adjust the structure by changing the winding orientation and position as shown in Fig.6.

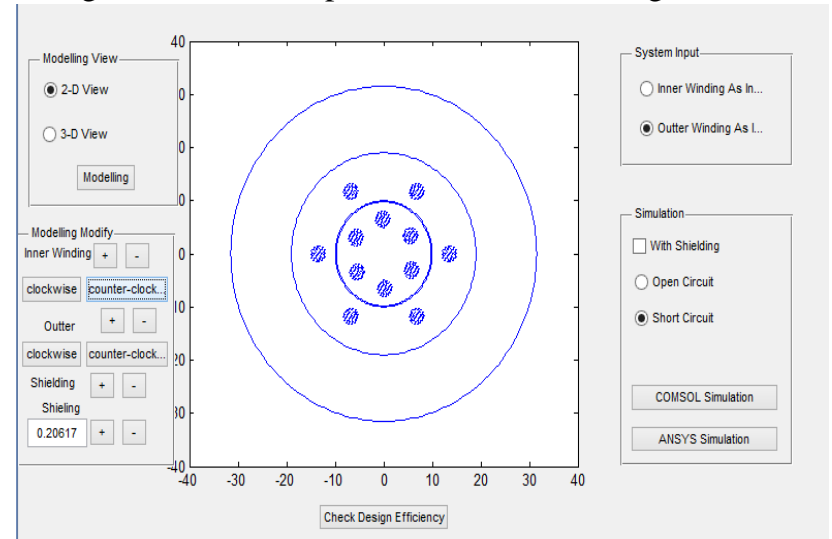

Fig. 6 Geometric Modelling for the FEM Simulation

In addition, in order to obtain FEM simulation results in different circumstances, the engineer can choose either an open circuit or a short circuit. The platform will generate a matrix which contains the ring core size, winding coordination, litz 
wire structure and the transformer's working parameters. The matrix will be sent to the FEM simulation software through the communication API and rebuild the modelling file. The simulation result can be obtained in the FEM simulation solver.

\section{VALIDATION AND VERIFICATION}

Example simulation results of the $8 \mathrm{~kW}$ HFCT are shown in Fig. 7 and Fig. 8. In Fig. 7, the flux distribution helps engineers to verify the effectiveness of designed structure. Based on the simulation result, the insertion of the Faraday shield has only minor negative effect on the magnetic performance of the HFCT. This negative effect is almost negligible; this is because the magnetic field is almost perpendicular to the shield conductor surface as well as the thin copper shield minimizing the side effect for the shielding insertion. On the other hand, the eddy current distribution simulation shown in Fig. 8 is very helpful on identifying the hot spots of the designed transformer, in that a heat sink can be fabricated to achieve better performance of heat dissipation. The simulation results shown in this paper are under the configuration of using the outer circular winding as the primary winding and the inner circular winding as its secondary winding.

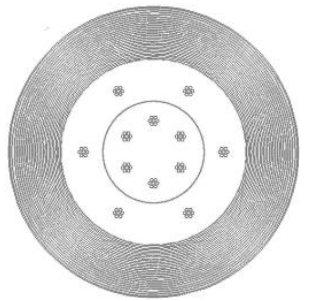

(a) Open Circuit (OC)

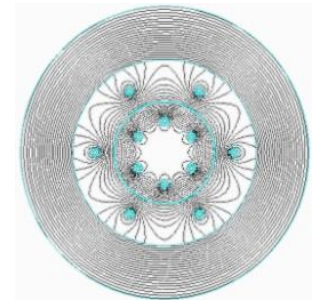

(b) Short Circuit (SC)
Fig. 7 Flux distribution of the 8 kW HFCT

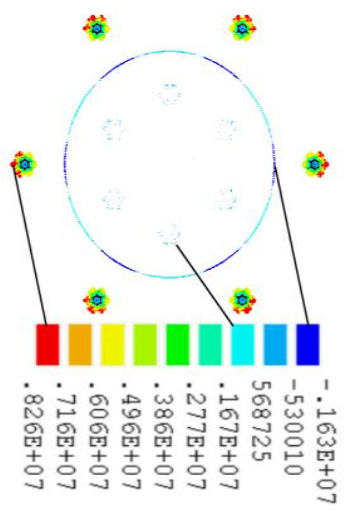

(a) Open Circuit (OC)

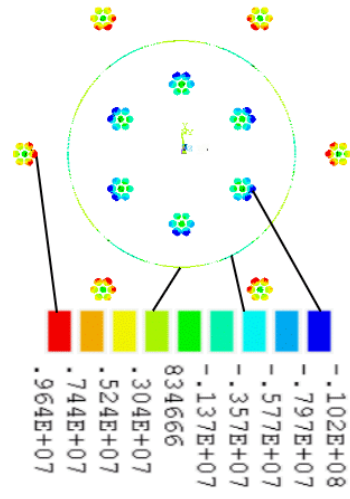

(b) Short Circuit (SC)
Fig. 8 Eddy current distribution of the $8 \mathrm{~kW}$ HFCT

Measurements of the prototype $8 \mathrm{~kW}$ HFCT is shown in table II, as well as the corresponding simulations. The measurement was conducted by using an $\mathrm{HP} 4285 \mathrm{~A}(75 \mathrm{kHz}$ to $30 \mathrm{MHz})$ precision LCR meter. $L_{s}$ and $R_{s}$ denote the leakage inductance and winding resistance respectively, $L_{p}$ denotes the magnetizing inductance, $R_{p}$ denotes the core loss resistance and $C_{p}$ denotes the intra-winding capacitance. A $0.7 \mu \mathrm{H}$ difference of $L_{s}$ is observed due to the mesh finesse, in which the result obtained requires a computation time of three hours based on a personal laptop. Also, there is a variation of $0.1 \Omega$ between the simulation and measurements of the $R_{s}$. The difference can mainly be attributed to the termination effect that the imperfect soldering increases the $R_{s}$ of the transformer. $L_{p}$ and $C_{p}$ are almost identical in both simulations and measurements; while $R_{p}$ is not applicable due to the non-linear characteristic of the magnetic material. In short, the coupling capacitance has been reduced by $77.13 \%$ with the Faraday shield inserted while only increases minor power losses.

TABLE II

COMPARISON OF IMPEDANCE RESULTS AT $100 \mathrm{KHZ}$ BETWEEN MEASUREMENTS AND SIMULATIONS

\begin{tabular}{lccccc}
\hline \hline & $L s(\mu \mathrm{H})$ & $R s(\Omega)$ & $L p(\mu \mathrm{H})$ & $R p(\mathrm{k} \Omega)$ & $C p(p \mathrm{~F})$ \\
\hline Measurement & 1.929 & 0.129 & 677.2 & 11.6 & 6.9 \\
Simulation & 1.287 & 0.022 & 679.6 & N/A & 7.93 \\
\hline
\end{tabular}

\section{CONCLUSION}

In this paper, an integrated HF transformer design platform is proposed and presented. It has been validated and proved by an $8 \mathrm{~kW}$ transformer design case study. The platform generates a coaxial transformer structure with a 21 -litz wire winding structure. The FEM simulation results indicate the transformer has been well designed and match with the real measurement result.

\section{REFERENCES}

[1] A. Halinka, M. Szewczyk, "ANN based detection of electrical faults in generator-transformer units," Eighth IEE International Conference on Developments in Power System Protection 2004, vol.1, pp.348-351, April 2004

[2] Z. Wang, Y. Liu, P.J. Griffin, "A combined ANN and expert system tool for transformer fault diagnosis," IEEE Power Engineering Society Winter Meeting, 2000, vol.2, pp.1261-1269 2000.

[3] D.Y. Shi, J. Buse, Q.H. Wu, L. Jiang, L, Y.S. Xue, "Fast identification of power transformer magnetizing inrush currents based on mathematical morphology and ANN," IEEE Power and Energy Society General Meeting, 2011, pp.1,6, July 2011.

[4] A.K. Yadav, A. Azeem, A. Singh, H. Malik and O.P. Rahi, "Application Research Based on Artificial Neural Network (ANN) to Predict No-Load Loss for Transformer's Design," Communication System and Network Technologies (CSNT), 2011 International Conference on, pp. 180, 183, June 2011.

[5] C. Nussbaum, H. Pfützner, Th. Booth, N. Baumgartinger, A. Ilo, and M. Clabian, "Neural Networks for the Prediction of Magnetic Transformer Core Characteristics," IEEE Transactions on Magnetics, vol. 36, no.1, January 2000.

[6] E. I. Amoiralis, P. S. Georgilakis, T. D. Kefalas, M. A. Tsili, and A. G. Kladas, "Artificial Intelligence Combined with Hybrid FEM-BE Techniques for Global Transformer Optimization," IEEE Transactions on Magnetics, vol. 43, no. 4, pp. 1633,1636, April 2007.

[7] W. Water and J. Lu, "Improved High-Frequency Planar Transformer for Line Level Control (LLC) Resonant Converters," IEEE Magnetics Letters, vol. 4, pp. 1-4, 2013.

[8] P. Zhou, W. N. Fu, D. Lin, S. Stanton and Z. J. Cendes, "Numerical modeling of magnetic devices," IEEE Transactions on Magnetics, vol. 40, no. 4, pp. 1803-1809, July 2004.

[9] P. S. Georgilakis and E. I. Amoiralis, "Spotlight on transformer design," IEEE Power and Energy Magazine, vol. 5, no. 1, pp. 40-50, Jan.Feb. 2007.

[10] W. Water and J. Lu, "Shielding Analysis of High-Frequency Coaxial Transformers Used for Electric Vehicle On-Board Charging Systems," IEEE Transactions on Magnetics, vol. 49, no. 7, pp. 4005-4008, July 2013.

[11] W.S. McCulloch and W. Pitts, "A Logical Calculus of the ideas immanent in nervous activity," Bulletin of Mathematical Biophysics, pp. 127-147, 1943 